\title{
Tectonic history of Europa: Coupling between internal evolution and surface stresses
}

\author{
Jun Kimura $^{1}$, Yasuko Yamagishi ${ }^{2}$, and Kei Kurita ${ }^{1}$ \\ ${ }^{1}$ Earthquake Research Institute, University of Tokyo, 1-1-1 Yayoi, Bunkyo-ku, Tokyo 113-0032, Japan \\ ${ }^{2}$ Institute for Research on Earth Evolution, Japan Agency for Marine-Earth Science and Technology, Yokosuka, Japan
}

(Received December 19, 2005; Revised October 3, 2006; Accepted October 4, 2006; Online published March 21, 2007)

\begin{abstract}
A stress history in the ice shell of Europa is presented. Europa's surface is ubiquitous in extensional tectonic features such as banded terrains. These surface features suggest that the surface may have been fractured and extended due to tensional stress, and various origins for such stresses have been proposed. We have focused on the solidification of the liquid water layer and the accompanying volume change as one of the dominant sources for such stresses. To estimate the stress state in the ice shell, we first performed numerical simulations of the thermal history. Based on the resulting structural evolution, we calculated stresses in the viscoelastic ice shell due to the solidification of the liquid layer. Europa's liquid layer solidifies slowly and may partially survive at present, and its solidification induces sufficient tensional stress to drive extensional tectonic activity. Consequently, we propose the tectonic scenario that the volume change due to phase change develops the basic amplitude of the stress, while tidal forces work as a trigger to fracture the surface.
\end{abstract}

Key words: Thermal history, tectonics, crustal stress, structural evolution.

\section{Introduction}

Some of the icy satellites display many surface features suggestive of a variety of tectonic activity. Recently, the Galileo spacecraft acquired many surface images of Jovian icy satellites at high resolution and revealed morphological characteristics of surface features. The most prominent feature of the icy satellites is an extensional fractured signature. One evident example is the banded terrain on Europa, consisting of straight or curved stripes, some of which are wedge-shaped. These features are interpreted as locations where the ice shell was fractured and dilated, then filled in with darker material at a later stage (Schenk and McKinnon, 1989; Pappalardo and Sullivan, 1996; Sullivan et al., 1998; Tufts et al., 2000; Prockter et al., 2002). Narrow ridges are also generally considered to be formed due to extensional fracturing (Greenberg et al., 1998). Europa's surface has been extensively modified by these ubiquitous tectonic structures. While these extensional features are present, compressional features or convergence regions compensating for surface extension have not been well-recognized (Prockter and Pappalardo, 2000; Greenberg, 2004). These characteristics suggest that the satellite has undergone a net global expansion. One of the most enigmatic problems associated with the icy satellites is the origin of this tectonic activity. The aim of this work is an evaluation of the tectonic stress induced due to phase changes in $\mathrm{H}_{2} \mathrm{O}$ and the accompanying volume changes.

Various origins for extensional stress have been proposed. The first one is solid state convection in the ice

Copyright (c) The Society of Geomagnetism and Earth, Planetary and Space Sciences (SGEPSS); The Seismological Society of Japan; The Volcanological Society of Japan; The Geodetic Society of Japan; The Japanese Society for Planetary Sciences; TERRAPUB. shell. As a driving force for tectonism, the generated tensional stress must exceed the mechanical strength of the ice shell, estimated to be of order $1 \mathrm{MPa}$ from experimental results (Hobbs, 1974; Gold, 1977). However, Squyres and Croft (1986) and McKinnon (1998) concluded that convective stress would be on the order of $0.01-0.1 \mathrm{MPa}$, which is too low to fracture the surface.

Tidal deformation may also be effective as a stress source. Surface tensional stress due to diurnal tidal deformation (Greenberg et al., 1998) and nonsynchronous rotation (Helfenstein and Parmentier, 1985; Leith and McKinnon, 1996) on Europa have been estimated to be about 0.1 $\mathrm{MPa}$ at a maximum. While these stress sources seem insufficient to induce surface fracture, there is plenty of evidence that orientations of bands and ridges are consistent with patterns estimated from theoretical models of tidal deformation (e.g., Greenberg et al., 1998). Therefore, no decisive conclusion as to the stress sources for tectonic activity has been obtained yet.

In terms of a volume change, three plausible causes are considered-differentiation, temperature change, and phase changes. Differentiation of $\mathrm{H}_{2} \mathrm{O}$ and the rocky component in the satellite that initially accreted as a homogeneous mixture possibly released a large amount of heat, yielding largescale melting; hence, traces of tectonism would have been erased. Thermal stress is also proposed as another source of volume expansion by Hiller and Squyres (1991), and they estimated the change in temperature in small-sized undifferentiated satellites. However, both processes should have occurred at an early stage, when the ice shell was incompletely formed and continuously disrupted by impacts of small bodies. Therefore, it is doubtful that these processes contributed to the volume expansion responsible for the ex- 


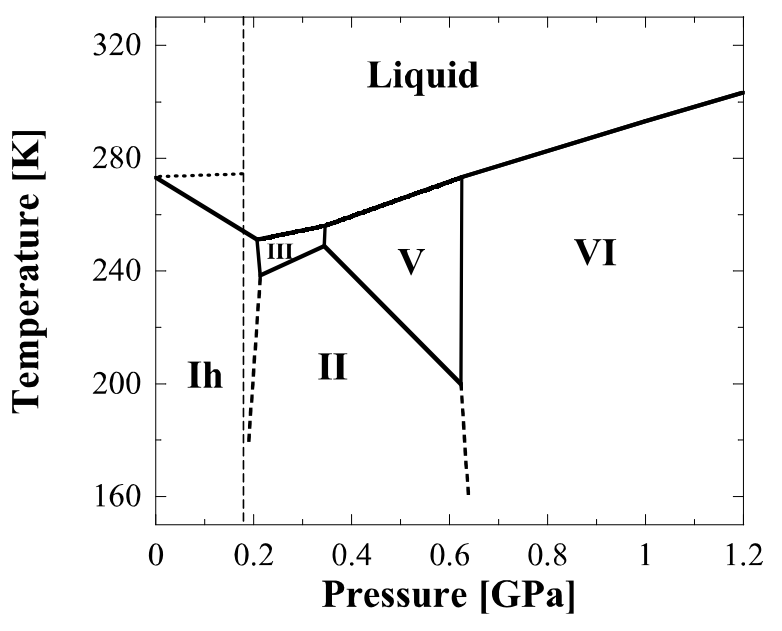

Fig. 1. Phase diagram of $\mathrm{H}_{2} \mathrm{O}$ in the pressure range 0-1.2 GPa. Dotted vertical line indicates pressure at the boundary between $\mathrm{H}_{2} \mathrm{O}$ layer and core for Europa. Dotted line indicates initial temperature for calculation of thermal history in Europa (adiabatic).

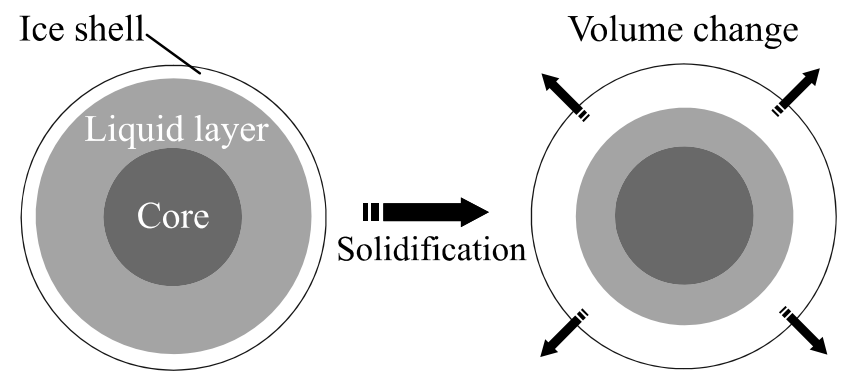

Fig. 2. Sketch of solidification of liquid $\mathrm{H}_{2} \mathrm{O}$ layer in Europa. As solidification of liquid layer and formation of ice Ih shell proceeds, a significant volume change arises. Satellite's surface tends to extend and to experience tensional hoop stress.

tensional features that we see at present. Zuber and Parmentier (1984) calculated the stress for Ganymede due to temperature change and the solid-solid phase change of ice. However, the solidification of the liquid layer was not considered, and the icy satellites should have had a liquid layer initially in their history.

Phase change (i.e., the solidification of liquid water) is another source of stress which may have contributed to the formation of extensional features on differentiated icy satellites. Icy satellites with a radius of more than several hundreds of kilometers should have achieved the release of accretional energy in the early stages of their history and so initially retain a liquid $\mathrm{H}_{2} \mathrm{O}$ layer (Lunine and Stevenson, 1982; Kuramoto and Matsui, 1994). Solidification of the liquid layer should have progressed with subsequent cooling, and the ice shell must have grown. The volume expansion due to the solidification of liquid water to low-pressure ice is up to $10 \%$. Although thermal contraction arises as the solid ice gets cooler (Nimmo, 2004), the effect of the solidification is much larger than that due to such a temperature change. Nevertheless, there have been few studies evaluating the stress in the ice shell and its time variation (see Fig. 2). An important point to note is that any evaluation of the stress history due to solidification of the liquid water layer is strongly coupled to the thermal history. Squyres
(1982) calculated the whole volume change of Ganymede due to solidification of the liquid layer. However, this evaluation was not based on the results of a thermal history. Moreover, relaxation of stress as well as its accumulation is inevitable because of the viscoelasticity of ice. Since the time scale for stress build-up due to the solidification is long, the ice shell should be treated as a viscoelastic material. Although Nimmo (2004) considered the ice-water volume change and calculated stresses in a viscoelastic shell, his evaluation of the process was not based on a thermal history.

In this work, the solidification rate of the liquid layer in Europa is determined by calculation of the thermal history, because the tectonic stresses generated in the ice shell due to solidification of the liquid layer strongly depend on the solidification rate. In other words, we must consider the coupling between the satellite's thermal history and its tectonic history. We treat only pure $\mathrm{H}_{2} \mathrm{O}$ as the material composing the outer layer of Europa in this work.

The goal of this paper is to evaluate the tectonic stress and discuss the scenario of tectonic activity on Europa. To attain the goal of this study, the following approach was used. Section 2 provides an outline of the thermal history model for estimating the solidification rate of the liquid layer as well as the concepts for calculation of generated stresses in the shell and explains the numerical procedure. In Section 3, we show the results for the solidification rate of the liquid layer and the tectonic stress induced due to solidification of the liquid layer. Finally, in Section 4, we discuss the role of stress sources for tectonic activity and the relation between the time scale for the relaxation of stress and the surface viscosity.

\section{Model}

\subsection{Solidification rate of the liquid water layer}

In order to evaluate the stress history, we need to constrain the solidification rate of the liquid water layer, which strongly depends on a viscous structure of the ice shell. The controlling parameter is the melting-point viscosity of ice $\eta_{0}$.

2.1.1 Initial structure and heat transfer model We assume that Europa has a radius $R_{s}=1565 \mathrm{~km}$ and a metalrock mixed core radius of $R_{c}=1400 \mathrm{~km}$. This structural model is consistent with the gravity data, and this data cannot distinguish between a differentiated core and an undifferentiated one (Anderson et al., 1998). In the initial state there is no solid ice shell, and the primitive liquid layer overlies the solid core. The temperature at the surface of the liquid layer is fixed at the zero-pressure melting temperature. Since the primitive liquid layer should vigorously convect due to rapid cooling from the surface, the liquid layer has an adiabatic temperature gradient. The core is initially isothermal with the temperature at the boundary with the overlying liquid layer. Such an initial structure of temperature is consistent with the model for the Galilean satellites formation (Lunine and Stevenson, 1982; Kuramoto and Matsui, 1994).

We consider that the mechanisms of heat transport in the satellites are convection and conduction. Radiation is neglected. The general equation of heat transfer is given as 
Table 1. Physical properties of core and of liquid layer.

\begin{tabular}{clccc}
\hline \multicolumn{1}{c}{ Layer } & \multicolumn{1}{c}{ Parameter } & Symbol & Unit & Value \\
\hline Liquid layer & Density & $\rho_{w}$ & $\mathrm{~kg} \mathrm{~m}^{-3}$ & 1000 \\
& Specific heat & $C_{p_{w}}$ & $\mathrm{~J} \mathrm{~K}^{-1} \mathrm{~kg}^{-1}$ & 4217 \\
& Thermal conductivity & $k_{c_{w}}$ & $\mathrm{~W} \mathrm{~m}^{-1} \mathrm{~K}^{-1}$ & 0.566 \\
& Thermal expansion coefficient & $\alpha_{w}$ & $\mathrm{~K}^{-1}$ & $2.1 \times 10^{-4}$ \\
& Viscosity & $\eta_{w}$ & $\mathrm{~Pa} \mathrm{~s}$ & $1.72 \times 10^{-3}$ \\
\multirow{3}{*}{ Core } & Density & $\rho_{c}$ & $\mathrm{~kg} \mathrm{~m}^{-3}$ & 3600 \\
& Specific heat & $C_{p_{c}}$ & $\mathrm{~J} \mathrm{~K}^{-1} \mathrm{~kg}^{-1}$ & 920 \\
& Thermal conductivity & $k_{c_{c}}$ & $\mathrm{Wm}^{-1} \mathrm{~K}^{-1}$ & 3.0 \\
& Thermal expansion coefficient & $\alpha_{c}$ & $\mathrm{~K}^{-1}$ & $2.4 \times 10^{-5}$ \\
\hline
\end{tabular}

follows:

$$
\rho C_{p} \frac{\partial T}{\partial t}=\nabla \cdot \mathbf{F}+\rho Q
$$

where $\rho$ is the density, $C_{p}$ is the specific heat, $\mathbf{F}$ is the heat flux, and $Q$ is the heat production rate. In the liquid water layer, we adopt parameterized convection theory to estimate convective heat flux and a simple energy balance without heat source. Thus, Eq. (1) yields

$$
\rho_{w} C_{p_{w}}\left(R_{s h}^{3}-R_{c}^{3}\right) \frac{d T_{l i q}}{d t}=3 R_{c}^{2} F_{B}-3 R_{s h}^{2} F_{T},
$$

where the suffix $w$ implies the liquid water, $R_{s h}$ is the position of the boundary between the ice shell and the liquid water layer. The heat fluxes at the top $F_{T}$ and the bottom $F_{B}$ of the liquid layer are obtained from the Nusselt numberRayleigh number relationship. We use the local Rayleigh number $R a_{l}$ and the local Nusselt number $N u_{l}$, which are defined by following equations (Honda, 1996),

$$
\begin{aligned}
& R a_{l} \equiv \frac{\rho \alpha g \Delta T_{l} D^{3}}{\kappa \eta} \\
& N u_{l} \equiv\left(\frac{R a_{l}}{R a_{l_{c}}}\right)^{\beta}
\end{aligned}
$$

where $\Delta T_{l}, D, \kappa$, and $\eta$ are the temperature difference in the top and bottom thermal boundary layer, the thickness of the liquid layer, the thermal diffusivity, and the viscosity, respectively. The suffix $l$ implies the local value ( $l$ is either $\mathrm{T}$ : top, or B: bottom). $R a_{l_{c}}$ is the critical Rayleigh number for the onset of convection, and it is about $10^{3} . \beta$ is a constant, and boundary layer theory gives a value of $1 / 3$; for turbulent convection ( $\left.R a_{l} \geq 10^{11}\right), \beta=2 / 7$ are used. Values of other parameters are summarized in Table 1. Heat fluxes of the top and bottom of the liquid layer are given by

$$
\begin{aligned}
& F_{l}=F_{\text {cond }} \cdot N u_{l} \\
& F_{\text {cond }}=k_{w} \frac{\Delta T_{l}}{D} .
\end{aligned}
$$

Heat transfer equation in the solid region, which are the ice shell and the core, is given by

$$
\begin{gathered}
\mathbf{F}=F_{\text {cond }}+F_{\text {conv }} \\
F_{\text {cond }}=k_{c} \nabla T \\
F_{\text {conv }}=k_{v}\left(\nabla T-\nabla_{a d} T\right)
\end{gathered}
$$

$$
\nabla_{a d} T=\left(\frac{\partial P}{\partial r}\right)\left(\frac{\partial T}{\partial P}\right)_{a d}=-\frac{\alpha g}{C_{p}} T,
$$

where $k_{c}$ is the thermal conductivity and $k_{v}$ is the effective thermal conductivity due to thermal convection. It is difficult to estimate the local Rayleigh number because viscosity of ice depends strongly on the temperature normalized by melting temperature, and the application of the parameterized convection theory is limited. Moreover, tidal dissipation acting as a heat source in the ice shell strongly depends on the local viscosity of ice. Therefore, we estimate convective heat flux $F_{\text {conv }}$ by the mixing length formulation extended to highly viscous fluids (Sasaki and Nakazawa, 1986; Abe, 1993). Thus, the effective thermal conductivity $k_{v}$ is given as follows:

$k_{v}= \begin{cases}0 & \frac{\partial T}{\partial r}<\left(\frac{\partial T}{\partial r}\right)_{a d} \\ \frac{\rho C_{p} \alpha g \ell^{4}}{18 v}\left[\frac{\partial T}{\partial r}-\left(\frac{\partial T}{\partial r}\right)_{a d}\right] & \frac{\partial T}{\partial r}>\left(\frac{\partial T}{\partial r}\right)_{a d}^{a}\end{cases}$

where $\ell$ is the mixing length and $v$ is the local kinematic viscosity. We take the mixing length $\ell$ as the distance from the nearest boundary of the convective layer. Values of parameters for the core are summarized in Table 1.

To calculate the solidification rate of the liquid water layer, we solve a moving boundary problem (Stefan problem). The equation for energy balance at the phase boundary is

$$
\rho_{s h} L \frac{d M_{i}}{d t}=F_{l i q}-F_{\text {sol }},
$$

where $\rho_{s h}$ is the density of the ice shell, $L$ is the latent heat, $M_{i}$ is the position of phase boundary, $F_{l i q}$ and $F_{\text {sol }}$ are the heat flow from the liquid layer and the solid layer, respectively. The temperature at the phase boundary is fixed at the melting point according to a pressure condition. We approximate the ice liquidus by the linear relation

$$
T_{m}=\frac{d T_{m}}{d P} P+T_{m_{0}},
$$

where $T_{m}$ is the melting temperature of ice, $P$ is the pressure and $T_{m_{0}}$ is $T_{m}$ at zero pressure. Values of parameters are summarized in Table 2.

The surface temperature is fixed at $100 \mathrm{~K}$ throughout the simulation (Ojakangas and Stevenson, 1989; Spencer et al., 1999). We consider heat generated by the decay of the longlived radioactive isotopes $\mathrm{U}, \mathrm{Th}$, and $\mathrm{K}$ in the core. The heat 
Table 2. Physical properties of ice Ih.

\begin{tabular}{lccc}
\multicolumn{1}{c}{ Parameter } & Symbol & Unit & Value \\
\hline Density $^{\mathrm{a}}$ & $\rho$ & $\mathrm{kg} \mathrm{m}^{-3}$ & 930 \\
Latent heat $^{\mathrm{a}}$ & $L$ & $\mathrm{~kJ} \mathrm{~kg}^{-1}$ & 284 \\
Melting temperature at $\mathrm{P}=0^{\mathrm{b}}$ & $T_{m 0}$ & $\mathrm{~K}$ & 273.2 \\
Slope of melting temperature & $d T_{m} / d P$ & $10^{-7} \mathrm{~K} \mathrm{~Pa}^{-1}$ & -1.063 \\
\hline${ }^{a}$ Hobbs (1974) & & & \\
${ }^{b}$ Sotin et al. $(1998)$ & & &
\end{tabular}

Table 3. Concentration, energy production, and decay constants of radiogenic elements.

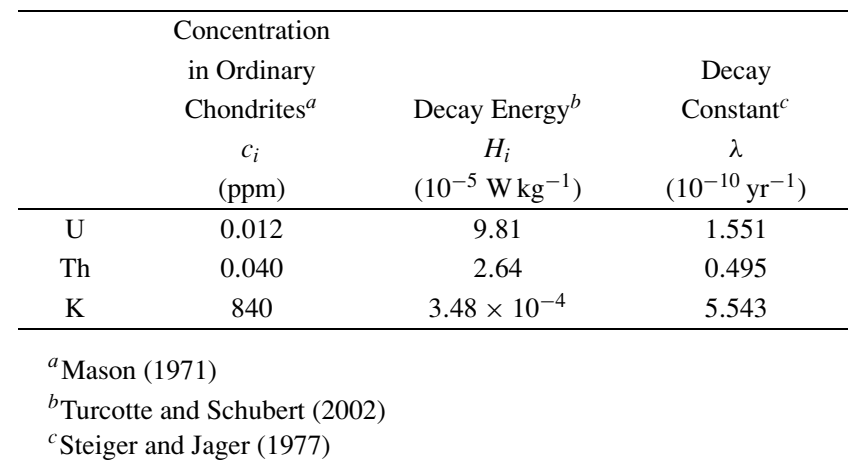

source term accompanying radioactive decay is described as follows:

$$
Q_{\text {radio }}=\sum c_{i} H_{i} \exp \left(-\lambda_{i} t\right),
$$

where $c_{i}$ is the concentration, $H_{i}$ is the heat release of the isotope, $\lambda_{i}$ is the decay constant, $i$ is the type of isotope, and $t$ is time before the present. These values are summarized in Table 3 (Schubert et al., 1986).

Within the ice shell, tidal heating is considered. The tidal heating rate in the solid core would be smaller than in the ice shell because it is mechanically decoupled from the ice shell by the liquid layer and, therefore, the core undergoes less deformation. We can evaluate the volumetric tidal heating rate using a Maxwell viscoelastic model (e.g., Segatz et al., 1988; Ojakangas and Stevenson, 1989). The average volumetric dissipation rate strongly depends on the temperature, and it can be calculated by (Showman and Han, 2004)

$$
Q_{\text {tide }}=\frac{\varepsilon_{0}^{2} \omega^{2} \eta_{s h}}{2\left[1+\frac{\omega^{2} \eta_{s h}^{2}}{\mu^{2}}\right]},
$$

where $\omega=2.05 \times 10^{-5} \mathrm{~s}^{-1}$ is the frequency of Europa's tidal flexing, and $\mu$ is the shear modulus of the ice shell. $\eta_{s h}$ is the temperature-dependent viscosity of the ice shell, which is determined later. $\varepsilon_{0}=\delta / R$ is the amplitude of tidal flexing strain during the tidal cycle, where $\delta$ is the amplitude of radial deflection due to tidal deformation and $R=1565 \mathrm{~km}$ is Europa's radius. For the generally accepted structure of Europa, which consists of the ice shell, the internal liquid layer, and the core, the peak radial deformation of the surface during a tidal cycle is about $30 \mathrm{~m}$ (Moore and Schubert, 2000). Although the amplitude of the tidal deformation depends on latitude and longitude, our simulations used $\varepsilon_{0}=1 \times 10^{-5}$ (corresponding to $\delta=16 \mathrm{~m}$ ) as a globally average value fixed in space and time. Finally, the tidal dissipation rate mainly depends on the viscosity profile in the ice shell.

Physical properties in the core are assumed to be constant except for the viscosity. We consider that the viscosity is temperature dependent and is described by

$$
\eta_{c}=\eta_{0_{c}} \exp \left[a\left(\frac{T_{m_{c}}}{T}\right)\right],
$$

where $\eta_{0_{c}}$ and $a$ are constants, and $T_{m_{c}}$ is the melting temperature. We adopt $\eta_{0_{c}}=4.9 \times 10^{8} \mathrm{~Pa} \mathrm{~s}, a=23.25$, and $T_{m_{c}}=1600 \mathrm{~K}$ (Karato et al., 1986).

In the ice shell, the temperature dependence of thermal properties is considered. Specific heat, thermal conductivity, and the thermal expansion coefficient are given by (Hobbs, 1974)

$$
\begin{gathered}
C_{p_{s h}}=7.037 T+185.0 \\
k_{c_{s h}}=\frac{488.12}{T}+0.4685 \\
\alpha_{s h}=3.0 \times\left(2.5 \times 10^{-7} T-1.25 \times 10^{-5}\right) .
\end{gathered}
$$

Heat transfer in the ice shell is greatly affected by the temperature dependence of viscosity, which is well approximated by the following equation

$$
\eta_{s h}=\eta_{0} \exp \left[A\left(\frac{T_{m}}{T}-1\right)\right] .
$$

The activation parameter $A$ is usually taken to be between 18 and 36 , which corresponds to an activation energy between about 40 and $80 \mathrm{~kJ}^{\text {mole }}{ }^{-1}$, and in this work we use $25 \mathrm{~kJ} \mathrm{~mole}^{-1}$ (Goldsby and Kohlstedt, 2001). As for the viscosity at the melting temperature $\eta_{0}$, we use a range of values for ice Ih between $10^{13}$ and $10^{15} \mathrm{~Pa}$. 


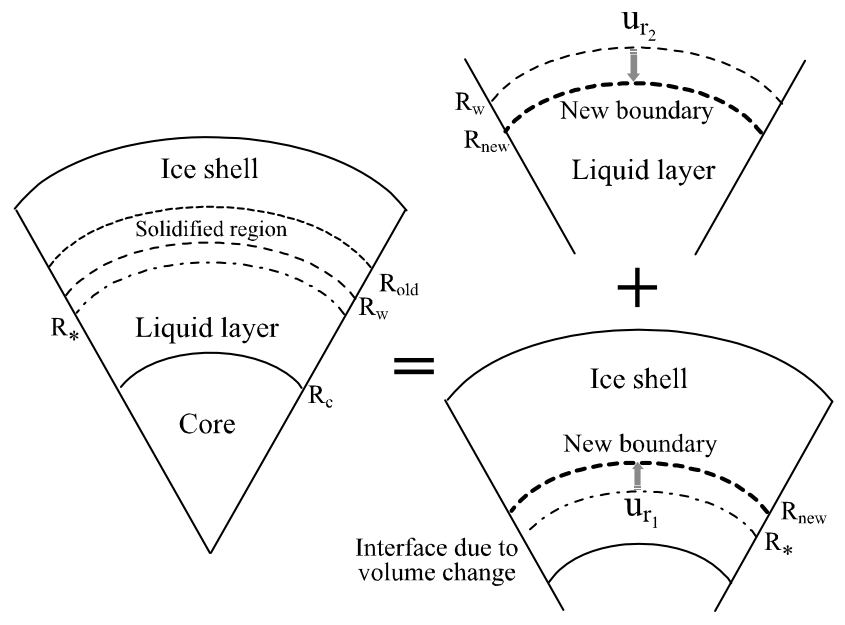

Fig. 3. Conceptual model for evaluation of stress generated during advance of phase boundary in Europa. $R_{\text {old }}$ is position of liquid-solid phase boundary. Shell $R_{\text {old }}-R_{w}$ is region undergoing phase change, as determined by Stefan's solution. Because of volume increase by factor of 1.09 during solidification, phase boundary under zero pressure moves to $R_{*}$. $R_{\text {new }}$ is position of new actual boundary as determined by equilibrium of pressure at the boundary.

Table 4. Parameters of elastic constants for the satellite.

\begin{tabular}{lcc}
\hline & $\begin{array}{c}\text { Bulk } \\
\text { modulus } \\
K(\mathrm{~Pa})\end{array}$ & $\begin{array}{c}\text { Shear } \\
\text { modulus } \\
\mu(\mathrm{Pa})\end{array}$ \\
\hline Core & $1.85 \times 10^{11}$ & $1.2 \times 10^{11}$ \\
Liquid layer & $2.0 \times 10^{9}$ & 0.0 \\
Ice shell & $1.0 \times 10^{10}$ & $3.3 \times 10^{9}$ \\
\hline
\end{tabular}

\subsection{Evaluation of the tectonic stress in the ice shell due to solidification of the liquid layer}

We assume that Europa is spherically symmetric and is composed of three concentric, homogeneous shells. Spherical coordinates $(r, \theta, \phi)$ are used with the origin at the satellite's center.

We firstly assume that each layer is perfectly elastic and has homogeneous properties. We consider the solidification of a liquid water layer occupying the region $R_{\text {old }}$ to $R_{w}$ in a unit time interval (Fig. 3). Because the liquid $\mathrm{H}_{2} \mathrm{O}$ increases its volume by a factor of 1.09 during solidification, the position of the bottom of the ice shell is no longer $R_{w}$. It must be located at an inner position $R_{*}$, which satisfies the mass balance equation,

$$
R_{*}^{3}=R_{\text {old }}^{3}-\frac{\rho_{l}}{\rho_{s}}\left(R_{\text {old }}^{3}-R_{w}^{3}\right),
$$

where $\rho_{l}$ and $\rho_{s}$ are the density of liquid and solid $\mathrm{H}_{2} \mathrm{O}$, respectively. Thus, the newly solidified ice would overlap the liquid layer. In response, the two phases are stressed to deform. One is a system in which the liquid layer pushes on the ice shell, and the other is a system in which the ice shell pushes back on the liquid layer (see Fig. 3). The place where both pressures balance should be the new actual boundary between the liquid layer and the ice shell $R_{\text {new }}$, and thus an excess pressure is generated. According to the equilibrium equations of elastic bodies (e.g., Landau and Lifshitz, 2000), the radial displacement $u_{r}(r)$ and the

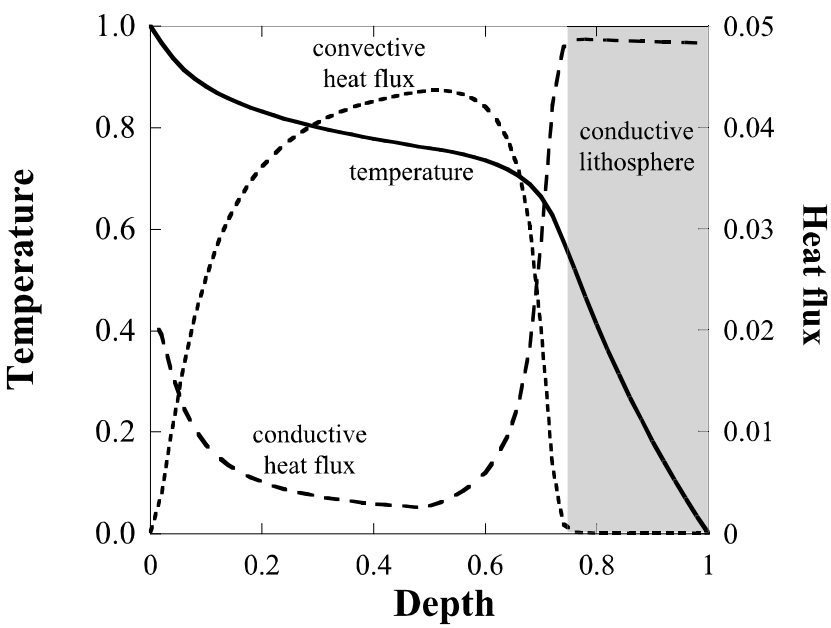

Fig. 4. Vertical profiles of nondimensionalized temperature (solid line, left axis), conductive (dashed line) and convective heat flux (dotted line, right axis) with in the ice shell for a typical snapshot of a numerical calculation of the thermal history. The thickness of the upper cold lithosphere which the tectonic stress concentrates is determined as a region where the convective heat flux becomes zero (gray region) (Davaille and Jaupart, 1994).

stress components $\sigma_{r r}(r), \sigma_{\theta \theta}(r)$ and $\sigma_{\phi \phi}(r)$ produced in each layer can be described (see Appendix). Our model considers the deformation of the liquid layer and the core. Elastic constants are summarized in Table 4. From the following boundary conditions

1) free surface $\sigma_{r r 1}(r)=0 \quad$ at $r=R_{s}$

2) normal stress $\sigma_{r r 1}(r)=-P_{l} \quad$ at $r=R_{*}$

3) normal stress $\sigma_{r r 2}(r)=-P_{s h} \quad$ at $r=R_{w}$

4) continuity $u_{r 2}(r)_{o c}=u_{r 2}(r)_{c} \quad$ at $r=R_{c}$

5) no displacement $u_{r 2}(r)_{c}=0 \quad$ at $r=0$,

where $P_{s h}$ and $P_{l}$ are the excess pressure at the upper boundary of the liquid layer and at the lower boundary of the ice shell, respectively, and $R_{c}$ is the radius of the solid core, we obtain the elastic solutions for $u_{r}(r), \sigma_{r r}(r)$, $\sigma_{\theta \theta}(r)$, and $\sigma_{\phi \phi}(r)$, which are given in the Appendix.

The excess pressures $P_{s h}$ and $P_{l}$ will balance as $P_{\text {new }}$ at the boundary between the liquid layer and the ice shell $R_{\text {new }}$. The deformation of each domain at the boundary is given by $u_{r_{1}}=R_{\text {new }}-R_{*}$ and $u_{r_{2}}=R_{\text {new }}-R_{w}$ (see Fig. 3). Using these equations and setting $P_{s h}=P_{l}=P_{\text {new }}$, the internal excess pressure is obtained as follows:

$$
P_{\text {new }}=\frac{R_{*}-R_{w}}{C_{1}-C_{2}-\left(C_{3} \cdot C_{4}\right)},
$$

where $C_{1}, C_{2}, C_{3}, C_{4}$, and the equilibrium position of the phase boundary $R_{\text {new }}$ are given in the Appendix.

When solid state convection develops in the ice shell, the conductive brittle layer termed lithosphere is formed in the upper cold region of the ice shell. Convective motion is restricted to a warmer sublayer below the lithosphere, termed the asthenosphere. To determine the lithospheric thickness, we evaluate the conductive and convective heat fluxes in the ice shell (Davaille and Jaupart, 1994). The bottom of the lithosphere is the depth at which the convective heat flux (a function of depth) becomes almost zero (Fig. 4). The position at which convection balances conduction should be 
the interface of the thermal boundary layer. We assume the asthenosphere behaves as a viscous fluid layer and that the internal excess pressure is supported by the lithosphere. In this convective case, new boundary conditions prescribing the continuity of radial stress and displacement are required.

Since we consider the solid layer to be a Maxwell medium, the viscoelastic solution can be obtained from the elastic expressions by the correspondence principle. We therefore make the substitution into the elastic solutions

$$
\mu_{s h} \rightarrow \tilde{\mu}(s)=\frac{\mu_{s h} s}{s+1 / \tau},
$$

where $s$ is the Laplace variable, $\tau=\eta_{s h} / \mu_{s h}$ is the characteristic Maxwell time, and $\eta_{s h}$ is the viscosity of the ice shell, which will be described later. We then introduce the step-like source function $P(t)=P_{\text {new }} H(t)$, giving the excess pressure in the liquid layer. According to the correspondence principle, we obtain the Laplace transform $\tilde{P}(s)=\tilde{P}_{\text {new }}(s) / s$, where $\tilde{P}_{\text {new }}(s)$ is described in the Appendix.

By substituting Eq. (22) into the elastic solution Eqs. (A.14) and (A.15), we obtain the viscoelastic solution of the converted problem in the $s$-space $\tilde{\sigma}_{r r}(s), \tilde{\sigma}_{\theta \theta}(s)$, and $\tilde{\sigma}_{\phi \phi}(s)$. Finally, using Laplace inversion we obtain the solutions for the tectonic stress components as functions of time;

$$
\begin{aligned}
\sigma_{r r}(r, t) & =\left(\frac{D}{R_{s}^{3}}-\frac{D}{r^{3}}\right) P_{\text {new }} \cdot \exp \left(-\frac{\delta}{\tau} t\right), \\
\sigma_{\theta \theta}(r, t) & =\sigma_{\phi \phi}(r, t) \\
& =\left(\frac{D}{R_{s}^{3}}-\frac{D}{2 r^{3}}\right) P_{\text {new }} \cdot \exp \left(-\frac{\delta}{\tau} t\right),
\end{aligned}
$$

where $D$ and $\delta$ are given in the Appendix.

The incremental model for stress evaluation in this work may underestimate the stress state in the ice shell, because a thin, stress-free shell is added during a unit time interval. However, this effect can be suppressed by making the time step sufficiently small.

Note that large uncertainty exists for the viscosity of the ice shell $\eta_{s h}$ when one considers the competition between the accumulation and relaxation of induced stresses due to viscoelastic behavior. Since the purpose of this work is to discuss the occurrence of tectonic activity at the surface, a viscosity value at the surface is necessary. Passey and Shoemaker (1982) calculated a surface viscosity for Ganymede of about $1 \times 10^{25} \mathrm{Pas}$, based on viscous relaxation of observational topography of impact craters. Because the Maxwell time calculated from this surface viscosity is about $100 \mathrm{Myr}$ and the growth of the ice shell is not so rapid-which will be shown next section-viscoelastic treatment is essentially important for this problem. On this basis, we use values from $10^{23}$ to $10^{27} \mathrm{~Pa}$ s for stress evaluation. In calculations of the thermal history, Eq. (19) for the viscosity of the ice shell are used and it yields a surface value of about $10^{32} \mathrm{~Pa}$ s using an expected surface temperature of $100 \mathrm{~K}$. This value is extremely large and seems to be an overestimation, while Eq. (19) is a good approximation of viscosity in the range of higher temperatures relatively near the melting point. However, in calculating the thermal

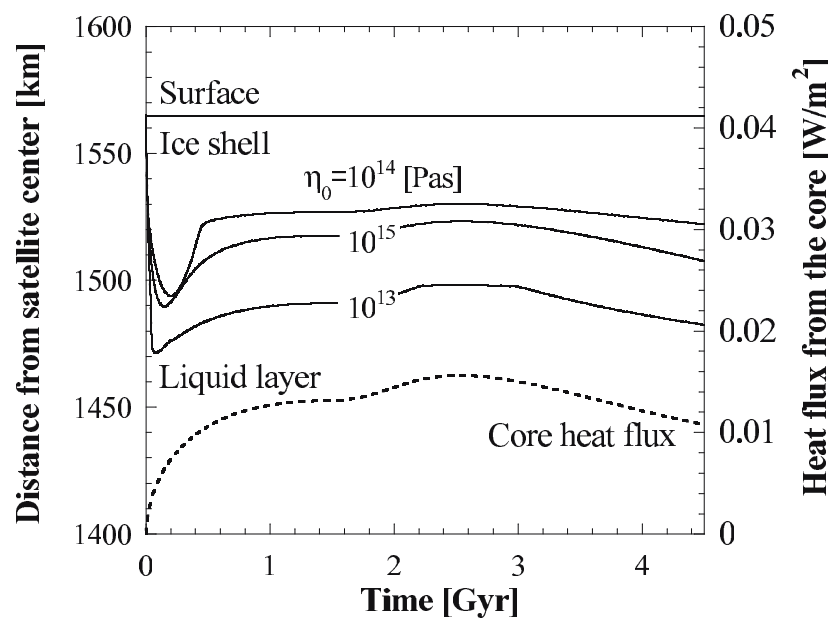

Fig. 5. Position of boundary between ice shell and liquid layer in Europa as function of time, for melting-point viscosity of ice $\eta_{0}$ of $10^{15}, 10^{14}$, and $10^{13} \mathrm{~Pa}$ s. Satellite's radius is $1,565 \mathrm{~km}$ and core radius is $1,400 \mathrm{~km}$. Heat flux from the core is also shown (dotted line) for the calculation using $\eta_{0}=10^{15} \mathrm{Pas}$. Even in case using other values of $\eta_{0}$, these have almost same trends.

history, differences in viscosity structure near the surface should not affect the heat transfer, and we use the viscosity structure which follows Eq. (19) through to the surface. This is because heat transport near the surface, where viscosity is large, is always controlled only by conduction, regardless of the exact viscosity.

\section{Results}

We begin by showing the solidification of the liquid layer in three cases of the melting point viscosity of ice $\eta_{0}$, followed by showing the evolution of surface stress for several values of the surface viscosities $\eta_{s}$. As last, we mention the thermal history of the core and discuss the effect of the initial temperature profile.

\subsection{Solidification of liquid layer}

Results of structural evolution are presented in Fig. 5 as a function of the melting point viscosity of ice $\eta_{0}$ (Eq. (19)) from $10^{13}$ to $10^{15} \mathrm{~Pa}$. The curves in these figures show the boundary between the ice shell and the liquid layer versus time, and temporal change of heat flux from the core is also shown. First, we discuss Fig. 5 for $\eta_{0}=10^{15} \mathrm{~Pa} \mathrm{~s}$, in detail. Snapshots of the temperature profiles in the ice shell and the core are shown in Figs. 6(a) and 7, respectively. The solidification scenario can be divided into the following stages:

(1) The liquid layer begins to solidify and the ice shell grows rapidly. Solidification continues until heat transport through the ice shell balances with heat flux from the core at $1.5 \times 10^{8}$ years. Heat transport in the ice shell is entirely dominated by conduction because the viscosity of the shell is relatively high and solid-state convection can hardly occur (Fig. 6(a)).

(2) The core gradually heats up due to radioactive decay, and the temperature at the core's surface begins to increase. Since the liquid layer convects and efficiently transports heat from the core to the ice shell, the ice shell is prevented from growing, and solidification of the liquid layer 

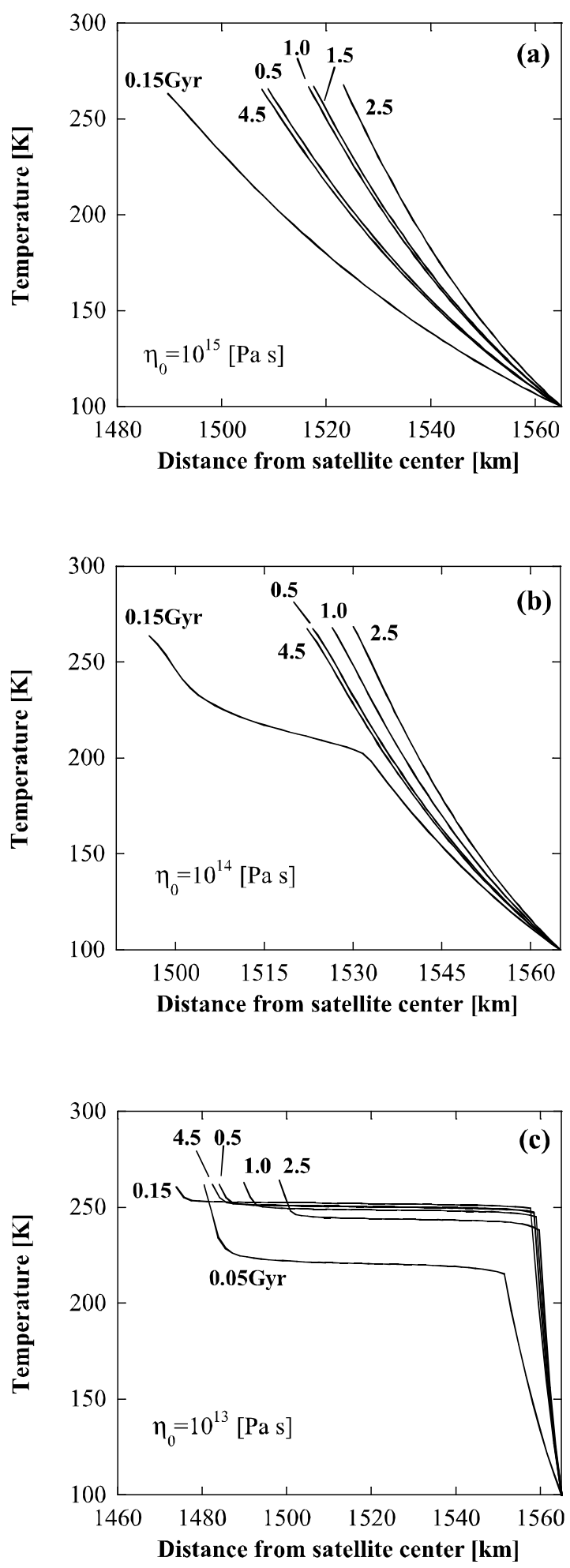

Fig. 6. Temperature profiles in ice shell of Europa (cf. Fig. 5), with melting-point viscosity of ice $\eta_{0}$ of $10^{15} \mathrm{~Pa} \mathrm{~s}(\mathrm{a}), 10^{14} \mathrm{~Pa} \mathrm{~s}(\mathrm{~b})$, and $10^{13}$ $\operatorname{Pas}(\mathrm{c})$.

ceases. Thereafter, radiogenic heating in the core continues to increase, and which leads to a thinner ice shell.

(3) In the core, when the temperature gradient becomes large, solid-state convection occurs at $1.6 \times 10^{9}$ years. Thereafter, the heat flux from the core into the liquid layer further increases, and the thickness of the ice shell further decreases.

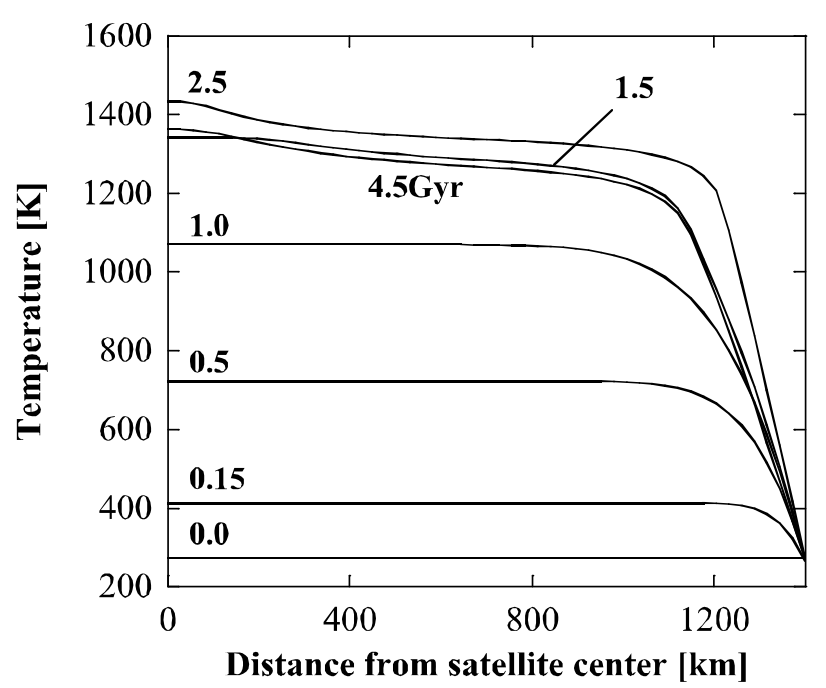

Fig. 7. Temperature profiles in the core of Europa, corresponding to Fig. 6(a) with melting-point viscosity of ice $\eta_{0}$ of $10^{15} \mathrm{Pas}$.

(4) The radiogenic heat source in the core becomes depleted, and the satellite enters a cooling stage at $2.5 \times 10^{9}$ years. The liquid layer begins gradually to solidify again.

Figure 5 indicates that a liquid layer with a thickness of $85-110 \mathrm{~km}$ is preserved at present under an ice shell that is $40-80 \mathrm{~km}$ thick. As the ice shell grows, the temperature difference through the liquid layer increases in accordance with a negative gradient of the melting curve of ice Ih, because the temperature at the bottom of the ice shell is fixed at the melting point. The liquid layer directly contacts the core and is heated due to heat generation in the core: therefore convection occurs in the liquid layer and heat from the core can be efficiently transported to the ice shell. In the ice shell, the heat transport is controlled only by conduction throughout the satellite's history (Fig. 6(a)), and the ice shell cannot so effectively remove the heat from inside. Therefore, solidification of the liquid layer proceeds very slowly in Europa.

The contribution of the tidal dissipation to this result is small because of the higher value of the melting-point viscosity of ice. The tidal dissipation rate depends on the thickness of the viscoelastic layer and on the temperature, and it reaches a maximum when the Maxwell time of ice approaches the orbital period. For this melting-point viscosity of ice, the Maxwell time at the base of the ice shell is similar to the orbital period of Europa. However, the temperature profile in the ice shell is purely conductive, so tidal heating is effective only at the very base of the ice shell. Consequently, tidal heating in the whole ice shell is small, and its volumetric rate is less than the radiogenic heating rate in the core by roughly an order of magnitude. In this work, the amplitude of tidal deformation is assumed to be about $16 \mathrm{~m}$, a relatively moderate value when compared to previous studies (e.g., Hussmann et al., 2002; Tobie et al., 2003). Note that if tidal heating is neglected, a liquid layer can survive until the present. For this melting-point viscosity of ice, the long lifetime of the liquid layer in Europa is achieved due to the efficiency of heat transfer through the ice shell rather than due to tidal heating. 

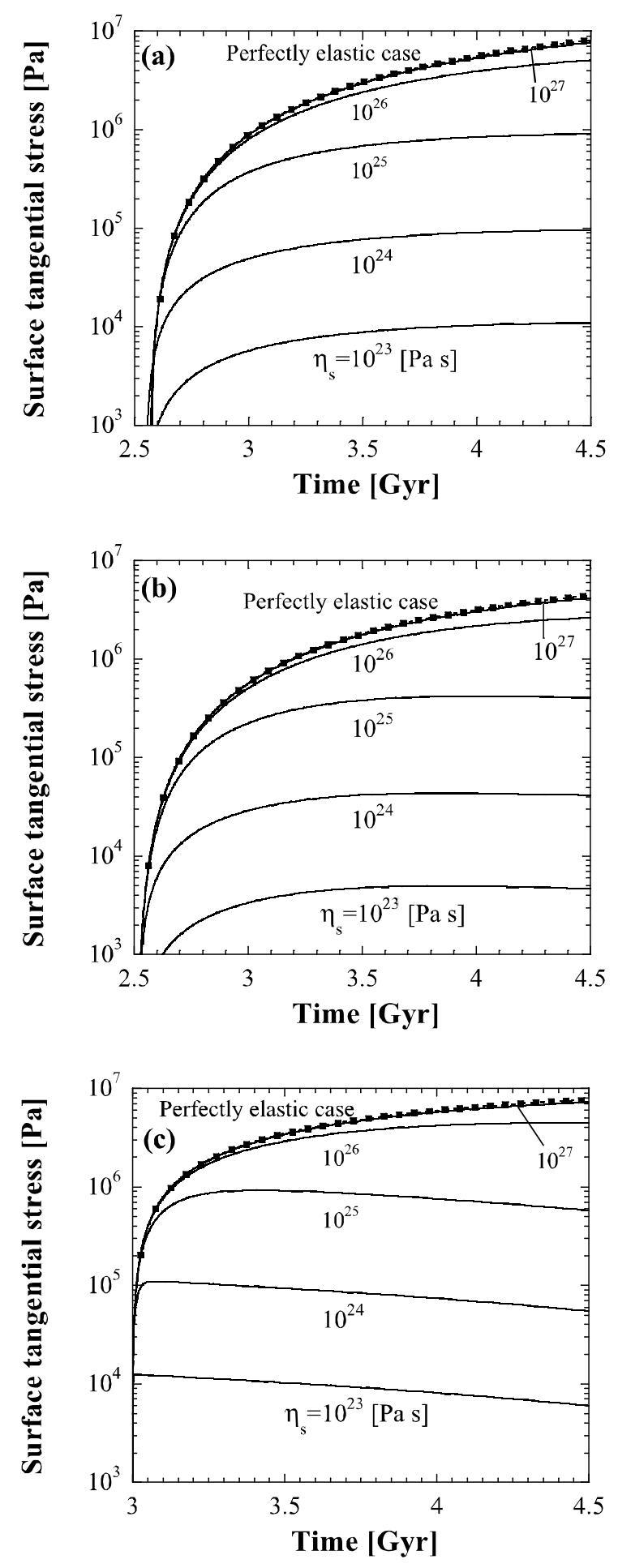

Fig. 8. Tangential stress (tension) at Europa's surface due to solidification of liquid layer and accompanying volume change, for melting-point viscosity of ice $\eta_{0}$ of $10^{15} \mathrm{Pas}(\mathrm{a}), 10^{14} \mathrm{Pas}(\mathrm{b})$, and $10^{13} \mathrm{Pas}$ (c). Amplitude of tensional stress is shown as a function of time and surface viscosity $\eta_{s}$. Stress is calculated for period in which Europa enters solidification stage of liquid layer, after about $2.5 \times 10^{9}$ years (a and b) and $3.0 \times 10^{9}$ years $(\mathrm{c})$. Dashed line with square-dots is perfectly elastic solution.

For a melting-point viscosity of $10^{14} \mathrm{~Pa} \mathrm{~s}$ (Fig. 5), although the overall evolution is similar to the previous case, the average thickness of the ice shell and the solidification rate are smaller. This is because the large dissipative re- gion in the ice shell is more widespread than in the case of $\eta_{0}=10^{15} \mathrm{~Pa} \mathrm{~s}$, and thus tidal heating in the ice shell is more effective. During solidification stage 1, solid-state convection occurs in the ice shell (Fig. 6(b)). While the efficiency of heat transfer through the ice shell increases due to convection, tidal heating is enhanced and the temperature gradient near the base of the ice shell diminishes. Therefore, the equilibrium thickness of the ice shell at the end of solidification stage 1 is not so different from the previous case. Even if tidal heating is neglected, the liquid layer can survive through to the present because the ice shell is largely stable with respect to solid-state convection throughout the satellite's history, as in the case of $\eta_{0}=10^{15} \mathrm{~Pa} \mathrm{~s}$, and thus the efficiency of heat transfer through the ice shell is not so effective.

In the case of $\eta_{0}=10^{13} \mathrm{Pas}$ (Fig. 5), the ice shell is largely unstable to solid-state convection. Although the rate of heat transfer through the ice shell is considerably larger and the ice shell becomes thicker than in previous cases, the tidal heating rate increases because of the warm convective asthenosphere in which the dissipation rate is large. The ice shell starts to convect when its whole thickness exceeds about $25 \mathrm{~km}$, and the convective asthenosphere and conductive lithosphere are clearly developed as the ice shell grows. Figure 6(c) shows that thermal boundary layers exist at the lower and upper regions of the ice shell. The conductive lithosphere cannot be discerned in Fig. 6(c), but it is distinguished from the upper thermal boundary layer by the definition described in the previous chapter. Tidal dissipation becomes intense in the region at the base of the ice shell through the convective asthenosphere, and the temperature of the asthenosphere increases. Because the large dissipative region will spread as the ice shell grows, the asthenosphere will get warmer and the lithosphere will become thinner. The temperature gradient at the base of the ice shell will decrease, and the efficiency of heat transport from inside will weaken. Consequently, the liquid layer is prevented from completely freezing. Note that if tidal heating is neglected altogether, the solidification of liquid layer will proceed very rapidly, and finally it will completely freeze during solidification stage 1 . Surface heat flows in the case of $\eta_{0}=10^{15}, 10^{14}$, and $10^{13} \mathrm{~Pa}$ are $0.01,0.015$, $0.07 \mathrm{~mW} / \mathrm{m}^{2}$, respectively.

If a melting-point viscosity is smaller than $10^{12} \mathrm{~Pa} \mathrm{~s}$, the liquid layer rapidly vanishes. In this case, the ice shell vigorously convects and removes heat from inside very rapidly. Tidal heating is weak because the viscosity is too low.

\subsection{Stress history of the ice shell}

Once the structural evolution is constructed based on the thermal history, we can evaluate the evolution of stress in the ice shell. Since the accumulated stress depends on the amount of incremental growth of ice in the viscoelastic medium, the controlling factor of the stress history is not the thickness of the ice shell but rather its changing rate. We particularly focus on the latest stage of solidification history because the average surface age of Europa, suggested from counting the number of impact craters, is quite young at 14-130 Myr (Zahnle et al., 2003, 2004). In the very early stages, even if the liquid layer solidifies very rapidly, thereby inducing significant volume changes, 
and shell stresses are induced, it should not contribute to the formation of the young surface features which we see at present. Moreover, for the nominal value of the surface viscosity of $10^{25} \mathrm{~Pa} \mathrm{~s}$, the Maxwell time is about $10^{8}$ years, and the changing rate of ice shell thickness is very small for hundreds of millions of years before the latest stage begins (see Fig. 5). Hence, the accumulated stress in the early stages should largely be relaxed by the onset of the latest solidification stage. If the surface viscosity is quite large, the stress accumulated in the early stages may persist, but this remains highly unclear because the ice shell must have been damaged by impacting bodies.

Figure 8(a) shows the time variation of tectonic stress at the surface, using the result of the solidification rate of the liquid layer for the melting-point viscosity of $10^{15} \mathrm{~Pa} \mathrm{~s}$ (see also Fig. 5). Viscosity at the surface $\eta_{s}$ is taken to be from $10^{23}$ to $10^{27} \mathrm{Pas}$. For large surface viscosities $\left(\eta_{s}=10^{26-27} \mathrm{~Pa} \mathrm{~s}\right)$, the stress in the ice shell is almost in the elastic regime, and it is largely unrelaxed. When the surface viscosity is small, the development of surface stress is more moderate. This is because it is easy for creep to arise in the ice shell, and the effect of stress relaxation overcomes that of stress accumulation. For this melting-point viscosity, the solidification rate of the liquid layer is initially small and increases quadratically with time (see Fig. 5, after 2.5 Gyr). Hence, the tectonic stress on the surface continues to develop under the conditions of all surface viscosities.

For evaluating the possibility of extensional tectonic activity, we should compare the strength of ice with the calculated tensional stress. If we adopt $1 \mathrm{MPa}$ as the tensile strength of ice, Fig. 8(a) shows that the generated tensional stress for the range of viscosities expected at the surface can exceed the tensile strength of ice. When the surface viscosity is small $\left(\eta_{s}<10^{25} \mathrm{~Pa} \mathrm{~s}\right)$, the generated surface stress is lower than the strength of ice. This is because the stress is relaxed over a time scale shorter than the solidification time scale.

Figure 8(b) shows the stress evolution for lower meltingpoint viscosity of ice, $\eta_{0}=10^{14} \mathrm{~Pa}$ s. Because the solidification rate is relatively small according to the calculation of thermal history, the generated stress is slightly smaller than for the case of $\eta_{0}=10^{15} \mathrm{~Pa}$. However, the overall stress history is similar to the previous case, and the stress generated for a range of expected surface viscosities can exceed the strength of ice.

Finally, in Fig. 8(c) we show the case of $\eta_{0}=10^{13} \mathrm{Pas}$. Solid-state convection occurs in the ice shell throughout the period of the stress calculation, and the lithosphere is formed near the surface (see Fig. 6(c)). The internal excess pressure is supported by the lithosphere, and the generated stress is concentrated in this layer. Since the solidification rate is getting smaller as time proceeds, stress relaxation becomes effective in an earlier stage and the tectonic stress begins to decrease for small surface viscosities. However, the generated stress is still sufficient to induce the tectonic activity.

All of these results for the thermal and structural evolution show that the subsurface liquid layer can be maintained throughout the satellite's history, and they are consistent with the general interpretation of the formation model of surface features and of induced magnetic field data (e.g., Khurana et al., 1998; Pappalardo et al., 1999; Kivelson et al., 2000). Moreover, we have shown that the tectonic stress generated at the surface due to solidification of the liquid layer is an adequate source for tectonic activity in Europa.

In order to evaluate the tectonic stress, we assumed an ice shell with homogeneous viscosity in this work. If we consider the structure of temperature-dependent viscosity in the actual ice shell, the excess pressure should disperse in the lower viscosity region, and the resultant tectonic stress in this deeper region may be a little smaller. However, the essential value for the discussion of surface fracturing is the surface stress which is calculated in the region of surface viscosity. Because the extent of such a surficial region seems to be thin compared with the whole ice shell and the internal excess pressure finally concentrates in this thin region, the induced surface stress should not be significantly depressed even if the pressure in deeper regions is somewhat smaller.

\subsection{Thermal history of the core}

Figure 7 shows the temporal change of temperature in the core corresponding to the case of $\eta_{0}=10^{15} \mathrm{Pas}$. The temperature in the core rises with time due to the decay energy of radio isotopes, and solid-state convection occurs at about 1.5 Gyr after formation of the satellite (see also dotted line in Fig. 5). Convection in the core starts in the upper part where the temperature gradient and the gravitational acceleration are sufficiently large, and the convective region gradually extends to the center as the temperature rises. In the cases of melting-point viscosities of $10^{14}$ and $10^{13} \mathrm{~Pa} \mathrm{~s}$, thermal evolution of the core follows a similar path.

In this work, the core starts from relatively cold state (Lunine and Stevenson, 1982). According to the gravitational energy associated with accretion, the initial core temperature might be warmer from tens or hundreds of K (Schubert et al., 1986). In this case until an initial warmer core would get rid of its heat, the ice shell would stay thin. However, because a timescale during which the core looses the initial heat is considerably short compared with the timescale required to enter the core's recent cooling stage, the thermal history follows the same trend as an initially cold case. Therefore, the initial thermal state of the core should hardly influence the thermal history and the growth rate of the ice shell after Europa enters the cooling stage.

\section{Discussions}

\subsection{Style of the tectonic activity on Europa: role of stress sources}

On Europa, the possible stress sources for extensional tectonic activity are tidal deformation, solid-state convection, and solidification of the liquid layer. The global distribution and the orientation of the major tectonic features are generally orthogonal to the directions of maximum tensional stress (Greenberg et al., 1998). These tectonic patterns possibly imply the role of non-synchronous rotation (Greenberg and Weidenschilling, 1984; Helfenstein and Parmentier, 1985; Geissler et al., 1998). On the other hand, the volume change which accompanies solidification of the liquid layer can provide an isotropic stress on the surface. Combining the surface observations indicative of tidal ori- 


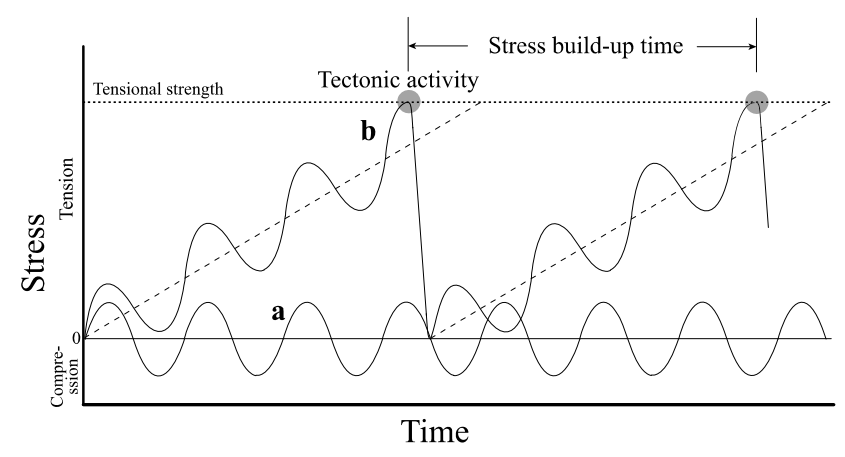

Fig. 9. Sketch of temporal change of tensional stress on Europa. Figure shows relationship between diurnal tidal stress (sinusoidal curve) and stress due to solidification of liquid water layer (dashed line) in schematic form. If only diurnal tidal stress acts on Europa's surface, its amplitude is not sufficient to induce extensional tectonic activity (a). We suggest that diurnal tidal stress is superimposed on stress due to solidification of the liquid layer (b), so that extensional tectonic activity occurs when the sum of these stresses exceeds the tensional strength of ice (dotted line). Time interval that the tensinal stress approaches strength of ice is defined as the stress build-up time.

gin and its insufficient amplitude, we propose the tectonic scenario in which the volume change due to phase change develops the basic amplitude of stress for surface tectonic activity, whereas the tidal forces work as a trigger to fracture and shape the surface. That is, the tectonic activity on the icy satellites is driven due to overlapping effects of these stress sources (Fig. 9).

If the surface features of Europa can be formed due only to tidal stress and/or solid-state convection, those surface activities should continue at present; changes in rotational history (e.g., variation of eccentricity) may also induce surface activity. However, such current geologic activity is not identified directly (Phillips et al., 2000). In other words, the present state may fall in a period in which the sum of the stresses does not exceed the strength of ice. There is a possibility that Europa entered the orbital resonance with Ganymede in past, and changes in rotational state (e.g., variation of eccentricity) may also induce surface tectonic activity. However, comparing a timescale for orbital change to that for resurfacing, the latter timescale is shorter in $\mathrm{Eu}-$ ropa based on the average surface age. Therefore, tectonic activities due to the past orbital resonances have not contributed to the present surface features.

When the sum will reach the critical amplitude, Europa will reenter an active period of global surface tectonics. The sequences of these processes will be repeated until the internal liquid layer completely solidifies. After that, the amplitude of the tidal deformation will be extremely small (Moore and Schubert, 2000), and the solid-state convection in the ice shell cannot contribute to surface manifestations (Rathbun et al., 1998). Thus, Europa may become inactive mechanically and thermally.

\subsection{Time for accumulation of stress, comparison with resurfacing time}

Here, we calculate the time required for accumulating tensional stresses to reach the tensile strength of ice. This time is called the stress build-up time (see, Fig. 9). We assume that when the amplitude of the generated stress

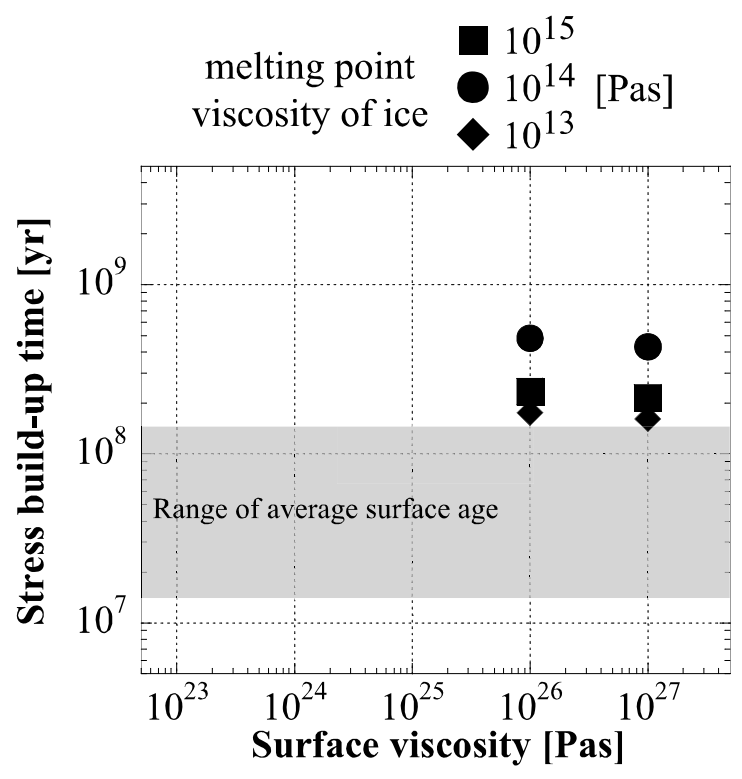

Fig. 10. Stress build-up time until tensional stress reaches the strength of ice (see also Fig. 9) is shown as function of surface viscosity. Using $\eta_{0}=10^{15} \mathrm{~Pa}$ s for solidification of the liquid layer, the required time for accumulating stress to reach the tensile strength of ice is about $2 \times 10^{8}$ years with $\eta_{s}=10^{26}$ or $10^{27} \mathrm{~Pa} \mathrm{~s}$ (squares). Results for $\eta_{0}=10^{14}$ (circle) or $10^{13} \mathrm{Pas}$ (diamond) as also shown. Shaded region shows range of average surface ages, 14-130 Myr, implied by crater counts (Zahnle et al., 2003, 2004).

reaches the strength of ice $(\sim 1 \mathrm{MPa})$, the stress is completely relaxed with the formation of extensional fractures. When the fractures are closed and the strength of the ice shell recovers, tectonic stresses will begin to accumulate again, and the satellite should experience a next cycle of tectonic processes. In other words, tectonic activity will occur after the stress build-up time. Figure 10 shows the average build-up time as a function of surface viscosity of $10^{23}$ to $10^{27} \mathrm{~Pa}$ s with $\eta_{0}=10^{13-15} \mathrm{~Pa}$. For example, when the melting-point viscosity of ice is $10^{15} \mathrm{Pas}$ and the surface viscosity is $10^{27} \mathrm{Pas}$, the build-up time is about $2 \times 10^{8}$ years. When $\eta_{s}<10^{25} \mathrm{~Pa} \mathrm{~s}$, no build-up time exists, because the tectonic stress cannot exceed the strength of ice (see Fig. 8(a)-(c)). The average surface age inferred by crater counting (Zahnle et al., 2003, 2004) is between 14 and $130 \mathrm{Myr}$, where this uncertainty is shown in Fig. 10.

If the surface viscosity is lower, the crater retention time becomes shorter and craters are easily relaxed. In the case of low surface viscosity, the actual surface age may be older than the cratering age because craters can be erased, even after surface activities cease. However, for example, the retention time of a $20 \mathrm{~km}$-diameter crater in the case $\eta_{s}=$ $10^{24} \mathrm{~Pa} \mathrm{~s}$ is estimated to be of the order $10^{9}$ years (Squyres and Croft, 1986), which is longer than the stress buildup time. Hence, the surface age should be controlled by the time scale of surface tectonic activity (e.g., extensional cracking and/or icy volcanism).

The average surface age is based on the estimate of impact rates and the paucity of craters on Europa, and this age represents the time which has passed since the last global resurfacing event occurred. The stress build-up times presented in Fig. 10 is longer than cratering age, because 
we assumed that the accumulated stress is completely relaxed when tectonic activity occurs, and thus the calculated model should be considered as an extreme case. Actually, accumulated stress should not be completely relaxed, so the strength of the ice shell will be recovered sooner and the tectonic activity should cease with little relaxation of stress. However, our model to calculate the stresses does not find the amount of stress relaxation during resurfacing activity, and there are no available publications on this subject. Hence, the resurfacing style of Europa's surface is not global but may be patchy. The stress build-up time in this case should be extremely shorter than that described in Fig. 10 and the total stress should keep a value close to the strength of ice, because the accumulated tectonic stress should not be completely relaxed by tectonic activity in reality.

To evaluate whether the style of tectonic activity is globally or patchy, the cross-cutting relationship and relative age between the tectonic features may help to solve this problem. Although such relations in regional scale have been discussed (e.g., Hoppa et al., 2001; Sarid et al., 2004), more detailed and globally scaled relationship from future observations will be needed.

\section{Concluding Summary}

As one of dominant stress sources for surface tectonic activities, we focused on the solidification of a liquid layer in icy satellites. In this work, we calculated the thermal and structural evolution of the icy satellites and evaluated the tectonic stress history due to solidification of the liquid $\mathrm{H}_{2} \mathrm{O}$ layer. In Europa, the subsurface liquid layer can only partially solidify and can survive until the present time for the various possible conditions of the melting-point viscosity of ice.

Using these results for the structural evolution, we evaluated the tectonic stress induced in the ice shell due to solidification of the liquid layer. We found the generated stress is sufficient to contribute to surface activity in comparison to the tensile strength of ice. Moreover, we proposed that surface tectonic activity is driven due to multiple effects of the stresses. The solidification of liquid water raises the basic amplitude of stress, and tidal deformation and/or solid-state convection in the ice shell creates the orientation and shape of surface features.

Acknowledgments. We are grateful to Kiyoshi Kuramoto and an anonymous reviewer for constructive comments. We also thank Craig R. Bina, whose suggestions improved the clarity of the descriptions very much. For this study, we have used the computer systems of the Earthquake Information Center of the Earthquake Research Institute, University of Tokyo.

\section{Appendix. Detailed description of the tectonic} stress

\section{Elastic case}

Here, we show the detailed descriptions of the tectonic stress due to the solidification of the liquid layer. Basic equations for the radial displacement $u_{r}(r)$ and the stress components $\sigma_{r r}(r), \sigma_{\theta \theta}(r)$ and $\sigma_{\phi \phi}(r)$ are given as follows
(Landau and Lifshitz, 2000):

$$
\begin{gathered}
u_{r}(r)= \begin{cases}a_{s h} r+\frac{b_{s h}}{r^{2}} & R_{*}<r<R_{s} \\
a_{l i q} r+\frac{b_{l i q}}{r^{2}} & 0<r<R_{w}\end{cases} \\
\sigma_{r r}(r)= \begin{cases}3 K_{s h} a_{s h}-4 \mu_{s h} \frac{b_{s h}}{r^{3}} & R_{*}<r<R_{s} \\
3 K_{l} a_{l}-4 \mu_{l} \frac{b_{l}}{r^{3}} & 0<r<R_{w}\end{cases}
\end{gathered}
$$

$$
\sigma_{\theta \theta}(r)=\sigma_{\phi \phi}(r)
$$

$$
= \begin{cases}3 K_{s h} a_{s h}+2 \mu_{s h} \frac{b_{s h}}{r^{3}} & R_{*}<r<R_{s} \\ 3 K_{l} a_{l}+2 \mu_{l} \frac{b_{l}}{r^{3}} & 0<r<R_{w}\end{cases}
$$

where $R_{s}$ is the radius of Europa, $K$ is the bulk modulus and $\mu$ is the shear modulus. $R_{*}$ and $R_{w}$ are described in Eq. (20) and Fig. 3. Suffixes $s h$ and $l$ indicate the ice shell and the liquid layer, respectively.

Coefficients $a_{s h}, b_{s h}, a_{l}$, and $b_{l}$ in Eqs. (A.1), (A.2), and (A.3) are given by

$$
\begin{gathered}
a_{s h}=\frac{D}{3 K_{s h} R_{s}^{3}} P_{l}, \\
b_{s h}=\frac{D}{4 \mu_{s h}} P_{l}, \\
a_{l}=-\frac{1}{3 K_{l}} P_{s h}, \\
b_{l}=\frac{R_{c}^{3}}{3 K_{l}} P_{s h}-\frac{R_{c}^{3}}{3 K_{c}} P_{l},
\end{gathered}
$$

and

$$
D=\frac{R_{*}^{3} R_{s}^{3}}{R_{s}^{3}-R_{*}^{3}},
$$

where $K_{c}$ is the bulk modulus of the solid core. The second term of the right-hand side in Eq. (A.7) represents a contribution of deformation of the core.

Expressions for $C_{1}, C_{2}, C_{3}$, and $C_{4}$ in Eq. (21) are given as follows:

$$
\begin{aligned}
C_{1} & =\frac{R_{c}^{3}-R_{w}^{3}}{3 K_{l} R_{w}^{2}}, \\
C_{2} & =\frac{R_{c}^{3}}{3 K_{c} R_{w}^{2}}, \\
C_{3} & =\frac{4 \mu_{s h} R_{*}^{3}+3 K_{s h} R_{s}^{3}}{R_{s}^{3}-R_{*}^{3}}, \\
C_{4} & =\frac{R_{*}}{12 K_{s h} \mu_{s h}} .
\end{aligned}
$$

The equilibrium position of the interface between the ice shell and the liquid layer $R_{\text {new }}$ yields

$$
R_{\text {new }}=\left(C_{1}-C_{2}\right) P_{n e w}+R_{w}
$$

Elastic components of the tectonic stress in the ice shell are given by

$$
\sigma_{r r}(r)=3 K_{s h} a_{n e w}-4 \mu_{s h} \frac{b_{n e w}}{r^{3}}
$$




$$
\sigma_{\theta \theta}(r)=\sigma_{\phi \phi}(r)=3 K_{s h} a_{n e w}+2 \mu_{s h} \frac{b_{n e w}}{r^{3}},
$$

where

$$
\begin{gathered}
a_{\text {new }}=\frac{D}{3 K_{s h} R_{s}^{3}} P_{\text {new }}, \\
b_{\text {new }}=\frac{D}{4 \mu_{s h}} P_{\text {new }} .
\end{gathered}
$$

That is, the elastic solutions of the tectonic stress in the ice shell can be described as functions of the excess pressure $P_{\text {new }}$.

\section{Viscoelastic case}

In order to obtain the viscoelastic solutions, we make substitution Eq. (A.16) and Eq. (A.17) into Eq (22). Then, $\tilde{a}_{\text {new }}(s)$ and $\tilde{b}_{\text {new }}(s)$ are given by

$$
\begin{gathered}
\tilde{a}_{\text {new }}(s)=\frac{D}{3 K_{s h} R_{s}^{3}} \tilde{P}_{\text {new }}(s), \\
\tilde{b}_{\text {new }}(s)=\frac{D}{4 \mu_{\text {sh }} s} \tilde{P}_{\text {new }}(s),
\end{gathered}
$$

where

$$
\tilde{P}_{\text {new }}(s)=\frac{R_{*}-R_{w}}{L},
$$

and

$$
\begin{aligned}
L= & C_{1}-C_{2} \\
& -\frac{\left[4 s \mu_{s h} R_{*}^{3}+3 K_{s h}(s+1 / \tau) R_{s}^{3}\right] R_{*}}{\left[R_{s}^{3}-R_{*}^{3}\right] 12 s K_{s h} \mu_{s h}} .
\end{aligned}
$$

$C_{1}$ and $C_{2}$ are defined in Eqs. (A.9) and (A.10).

Using the inverse Laplace transform and rearranging equations, we finally obtain the viscoelastic solution for the tectonic stress in the ice shell described in Eqs. (23) and (24). The term $\delta$ in Eqs. (23) and (24) is given by

$$
\delta=\frac{\frac{D}{4 R_{*}^{2}}}{\frac{D}{4 R_{*}^{2}}-\mu_{s h} B},
$$

where

$$
B=C_{1}-C_{2}-\frac{R_{*}}{3 K_{s h} R_{s}^{3}} D .
$$

\section{References}

Abe, Y., Thermal evolution and chemical differentiation of the terrestrial magma ocean, in Evolution of the Earth and Planets, edited by E. Takahashi, R. Jeanloz, and D. Rubie, 159 pp., American Geophysical Union, Washington DC, 1993.

Anderson, J. D., G. Schubert, R. A. Jacobson, E. L. Lau, W. B. Moore, and W. L. Sjogren, Europa's differentiated internal structure: Inferences from four Galileo encounters, Science, 281, 2019-2022, 1998.

Davaille, A. and C. Jaupart, Onset of thermal convection in fluids with temperature-dependent viscosity: Application to the oceanic mantle, $J$. Geophys. Res., 99, 19853-19866, 1994.

Geissler, P. E., R. Greenberg, G. Hoppa, P. Helfenstein, A. McEwen, R. T. Pappalardo, B. R. Tufts, M. Ockert-Bell, R. Sullivan, R. Greeley, M. J. S. Belton, T. Denk, B. Clark, J. Burns, J. Veverka, and the Galileo Imaging Team, Evidence for non-synchronous rotation of Europa. $\mathrm{Na}$ ture, 391, 368-370, 1998.

Greenberg, R., The evil twin of Agenor; Tectonic convergence on Europa, Icarus, 167, 313-319, 2004
Greenberg, R. and S. J. Weidenschilling, How fast do Galilean satellites spin?, Icarus, 58, 186-196, 1984.

Greenberg, R., P. Geissler, G. V. Hoppa, B. R. Tufts, D. D. Durda, R. Pappalardo, J. W. Head, R. Greeley, R. Sullivan, and M. H. Carr, Tectonic processes on Europa: Tidal stresses, mechanical response, and visible features, Icarus, 135, 64-78, 1998.

Gold, L. W., Engineering properties of fresh-water ice, J. Glaciology, 19, 197-223, 1977.

Goldsby, D. L. and D. L. Kohlstedt, Superplastic deformation of ice: Experimental observations, J. Geophys. Res., 106, 11017-11030, 2001.

Helfenstein, P. and E. M. Parmentier, Patterns of fracture and tidal stresses due to nonsynchronous rotation: Implications for fracturing on Europa, Icarus, 61, 175-184, 1985.

Hiller, J. and S. W. Squyres, Thermal stress tectonics on the satellites of Saturn and Uranus, J. Geophys. Res., 96, 15665-15674, 1991.

Hobbs, P. V., Ice Physics, Oxford University Press, London, 1974.

Honda, S., Local Rayleigh and Nusselt numbers for cartesian convection with temperature-dependent viscosity, Geophys. Res. Lett., 23, 24452448, 1996.

Hoppa, G. V., B. R. Tufts, R. Greenberg, T. A. Hurford, D. P. O’Brien, and P. E. Geissler, Europa's rate of rotation derived from the tectonic sequence in the Astypalaea region, Icarus, 153, 208-213, 2001.

Hussmann, H., T. Spohn, and K. Wieczerkowski, Thermal equilibrium states of Europa's ice shell: Implications for internal ocean thickness and surface heat flow, Icarus, 156, 143-151, 2002.

Karato, S., M. S. Peterson, and J. D. FitzGerald, Rheology of synthetic olivine aggregates: Influence of grain size and water, J. Geophys. Res., 91, 8151-8176, 1986.

Khurana, K. K., M. G. Kivelson, D. J. Stevenson, G. Schubert, C. T. Russell, R. J. Walker, and C. Polanskey, Induced magnetic field as evidence for subsurface oceans in Europa and Callisto, Nature, 395, 777-780, 1998 .

Kivelson, M. G., K. K. Khurana, C. T. Russell, M. Volwerk, R. J. Walker, and C. Zimmer, Galileo magnetometer measurements: A stronger case for a subsurface ocean at Europa, Science, 289, 1340-1343, 2000.

Kuramoto, K. and T. Matsui, Formation of a hot proto-atmosphere on the accreting giant-icy-satellite: Implications for the origin and evolution of Titan, Ganymede and Callisto, J. Geophys. Res., 99, 21183-22120, 1994

Landau, L. and E. Lifshitz, Theory of Elasticity, 3rd ed., Pergamon Press, Oxford, 2002

Leith, A. C. and W. B. McKinnon, Is there evidence for polar wander on Europa?, Icarus, 120, 387-398, 1996.

Lunine, J. and D. J. Stevenson, Formation of the Galilean satellites in a gaseous nebula, Icarus, 52, 14-39, 1982.

Mason, B., Handbook of Elemental Abundances in Meteorites, New York, Gordon and Breach, 1971.

McKinnon, W. B., Geodynamics of icy satellites, in Solar System Ices, edited by B. Schmitt et al., pp. 525-550, Kluwer Academic Press, Dordrecht, 1998.

Moore, W. B. and G. Schubert, Note: The tidal response of Europa, Icarus, 147, 317-319, 2000.

Nimmo, F., Stresses generated in cooling viscoelastic ice shells: Application to Europa, J. Geophys. Res., 109, doi:10.1029/2004JE002347, 2004.

Ojakangas, G. W. and D. J. Stevenson, Thermal state of an ice shell on Europa, Icarus, 81, 220-241, 1989.

Pappalardo, R. T. and R. J. Sullivan, Evidence for separation across a gray band on Europa, Icarus, 123, 557-567, 1996.

Pappalardo, R. T., M. J. S. Belton, H. H. Breneman, M. H. Carr, C. R. Chapman, G. C. Collins, T. Denk, S. Fagents, P. E. Geissler, B. Giese, R. Greeley, R. Greenberg, J. W. Head, P. Helfenstein, G. Hoppa, S. D. Kadel, K. P. Klaasen, J. E. Klemaszewski, K. Magee, A. S. McEwen, J. M. Moore, W. B. Moore, G. Neukum, C. B. Phillips, L. M. Prockter, G. Schubert, D. A. Senske, R. J. Sullivan, B. R. Tufts, E. P. Turtle, R. Wagner, and K. K. Williams, Does Europa have a subsurface ocean? Evaluation of the geological evidence. J. Geophys. Res., 104, 2401524055, 1999.

Passey, Q. R. and E. M. Shoemaker, Craters and basins on Ganymede and Callisto: Morphological indicators of crustal evolution, in Satellites of Jupiter, edited by D. Morrison, pp. 379-434, Univ of Arizona Press, Tucson, 1982

Phillips, C. B., A. S. McEwen, G. V. Hoppa, S. A. Fagents, R Greeley, J. E. Klemaszewski, R. T. Pappalardo, K. P. Klaasen, and H. H. Breneman, The search for current geologic activity on Europa, J. Geophys. Res., 105, 22579-22597, 2000. 
Prockter, L. M. and R. T. Pappalardo, Folds on Europa: Implications for crustal cycling and accommodation of extension, Science, 289, 941944, 2000.

Prockter, L. M., J. W. Head, R. T. Pappalardo, R. J. Sullivan, A. E. Clifton, B. Giese, R. Wagner, and G. Neukum, Morphology of Europan bands at high resolution: A mid-ocean ridge-type rift mechanism, J. Geophys. Res., 107, doi:10.1029/2000JE001458, 2002.

Rathbun, J. A., G. S. Musser, and S. W. Squyres, Ice diapirs on Europa: Implications for liquid water, Geophys. Res. Lett., 25, 4157-4160, 1998.

Sarid, A. R., R. Greenberg, G. V. Hoppa, P. Geissler, and B. Preblich, Crack azimuths on Europa: Time sequence in the sourthern leading face, Icarus, 168, 144-157, 2004.

Sasaki, S. and K. Nakazawa, Metal-silicate fractionation in the growing Earth: Energy source for the terrestrial magma ocean, J. Geophys. Res., 91, 9231-9238, 1986.

Schenk, P. and W. B. McKinnon, Fault offsets and lateral crustal movement on Europa: Evidence for a mobile ice shell, Icarus, 79, 75-100, 1989.

Schubert, G., T. Spohn, and R. T. Reynolds, Thermal histories, and internal structures of the moons of the solar system, in Satellites, edited by J. A. Burns and M. S. Matthews, pp. 224-292, Univ of Arizona Press, Tucson, 1986.

Segatz, M., T. Spohn, M. N. Ross, and G. Schubert, Tidal dissipation, surface heat flow, and figure of viscoelastic models of Io, Icarus, 75, 187-206, 1988.

Showman, A. P. and L. Han, Numerical simulations of convection in Europa's ice shell: Implications for surface features, J. Geophys. Res., 109, doi:10.1029/2003JE002103, 2004.

Sotin, C., O. Grasset, and S. Beauchesne, Thermodynamic properties of high pressure ices: Implications for the dynamics and internal structure of large icy satellites, in Solar System Ices, edited by B. Schmitt et al., pp. 79-96, Kluwer Academic Press, Dordrecht, 1998.

Spencer, J. R., L. K. Tamppari, T. Z. Martina, and L. D. Travis, Temperatures on Europa from Galileo photopolarimeter-radiometer: Nighttime thermal anomalies, Science, 284, 1514-1516, 1999.
Squyres, S. W., The evolution of tectonic features on Ganymede, Icarus, 52, 545-559, 1982.

Squyres, S. W. and K. C. Croft, The tectonics on icy satellites, in Satellites, edited by J. A. Burns and M. S. Matthews, pp. 293-341, Univ of Arizona Press, Tucson, 1986.

Steiger, R. H. and E. Jager, Subcommission on geochronology: Convention on the use of decay constants in geo- and cosmo-chronology, Earth Planet. Sci. Lett., 36, 359-362, 1977.

Sullivan, R., R. Greeley, K. Homan, J. Klemaszewski, M. J. S. Belton, M. H. Carr, C. R. Chapman, R. Tufts, J. W. Head, R. T. Pappalardo, J. Moore, P. Thomas, and the Galileo Imaging Team, Episodic plate separation and fracture infill on the surface of Europa, Nature, 391, 371373, 1998.

Tufts, B. R., R. Greenberg, G. Hoppa, and P. Geissler, Lithospheric dilation on Europa, Icarus, 146, 75-97, 2000.

Tobie, G., G. Choblet, and C. Sotin, Tidally heated convection: Constraints on Europa's ice shell thickness, J. Geophys. Res., 108, doi:10.1029/2003JE002099, 2003.

Turcotte, D. L. and G. Schubert, Geodynamics, 2nd ed., John Wiley, New York, 2002.

Zahnle, K., P. Schenk, H. Levison, and L. Dones, Cratering rates in the outer solar system, Icarus, 163, 263-289, 2003.

Zahnle, K., P. Schenk, L. Dones, and H. Levison, Cratering rates in the Jovian system, Workshop on Europa's icy shell: Past, Present, and Future, abstract 7052, 2004.

Zuber, M. T. and E. M. Parmentier, Lithospheric stress due to radiogenic heating of an ice-silicate body: Implications for Ganymede's tectonic evolution, Proc. Lunar Planet. Sci. Conf. 14th., in J. Geophys. Res. Suppl. 89, B429-B437, 1984.

J. Kimura (e-mail: junkim@eri.u-tokyo.ac.jp), Y. Yamagishi, and K. Kurita 